\title{
Orbital magnetization and its effects in spin-chiral ferromagnetic kagomé lattice
}

\author{
Zhigang Wang and Ping Zhang \\ Institute of Applied Physics and Computational Mathematics, P.O. Box 8009, Beijing 100088, P.R. China
}

\begin{abstract}
Recently, Berry phase in the semiclassical dynamical of Bloch electrons has been found to make a correction to the phase-space density of states and a general multi-band formula for finitetemperature orbital magnetization has been given [Phys. Rev. Lett. 97, 026603 (2006)], where the orbital magnetization $\mathcal{M}$ consists of two parts, i.e., the conventional part $M_{c}$ and the Berry-phase correction part $M_{\Omega}$. Using this general formula, we theoretically investigate the orbital magnetization and its effects on thermoelectric transport and magnetic susceptibility properties of the two-dimensional kagomé lattice with spin anisotropies included. The study in this paper is highly interesting by the occurrence of nonzero Chern number in the lattice. The spin chirality parameter $\phi$ (see text) results in profound effects on the orbital magnetization properties. It is found that the two parts in orbital magnetization opposite each other. In particular, we show that $M_{c}$ and $M_{\Omega}$ yield the paramagnetic and diamagnetic responses, respectively. It is further shown that the orbital magnetization displays fully different behavior in the metallic and insulating regions, which is due to the different roles $M_{c}$ and $M_{\Omega}$ play in these two regions. The anomalous Nernst conductivity is also calculated, which displays a peak-valley structure as a function of the electron Fermi energy.
\end{abstract}

PACS numbers: 75.30.-m, 73.43.-f, 72.15.Jf

\section{INTRODUCTION}

In the semiclassical dynamics of Bloch electrons, the Liouville's theorem on the conservation of phase-space volume is violated by the occurrence of Berry phase, which leads to a modification of the phase-space density of states 1 . This modified phase-space density of states, $D_{n}(\mathbf{r}, \mathbf{k})=1+e \mathbf{B} \cdot \boldsymbol{\Omega}_{n} / \hbar$, enters naturally in the semiclassical expression for the expectation value of physical quantities, and has profound effects on equilibrium as well as transport properties. Here $\mathbf{B}$ is the external magnetic field, and $\boldsymbol{\Omega}_{n}(\mathbf{k})$ is the Berry curvature (gauge flux) of electronic Bloch states defined by $\boldsymbol{\Omega}_{n}(\mathbf{k})=i\left\langle\nabla_{\mathbf{k}} u_{n}(\mathbf{k})|\times| \nabla_{\mathbf{k}} u_{n}(\mathbf{k})\right\rangle$ with $\left|u_{n}(\mathbf{k})\right\rangle$ being the periodic part of Bloch wave for the $n$-th band. Based on this correction in the semiclassical phase-space density, Xiao et al $\stackrel{2}{2}$ have further derived a general expression for the single-particle free energy in the presence of the external magnetic field as follows (for a general three dimensional system)

$$
F=-\frac{1}{\beta} \sum_{n} \int d^{3} \mathbf{k}\left(1+\frac{e}{\hbar} \mathbf{B} \cdot \boldsymbol{\Omega}_{n}(\mathbf{k})\right) \ln \left[1+e^{\beta\left(\mu-\varepsilon_{n}^{M}\right)}\right] .
$$

Here $\mu$ is the electron chemical potential, $\beta=1 / k_{B} T$, and $\varepsilon_{n}^{M}=\varepsilon_{n \mathbf{k}}-\mathbf{m}_{n}(\mathbf{k}) \cdot \mathbf{B}$ is the electron band energy in the presence of the external magnetic field. $\mathbf{m}_{n}(\mathbf{k})$ is the crystal orbital magnetic moment and conventionally defined by $\stackrel{3.4}{\underline{4}} \mathbf{m}_{n}(\mathbf{k})=-i(e / 2 \hbar)\left\langle\nabla_{\mathbf{k}} u_{n}(\mathbf{k})\right| \times[\hat{H}(\mathbf{k})-$ $\left.\varepsilon_{n}(\mathbf{k})\right]\left|\nabla_{\mathbf{k}} u_{n}(\mathbf{k})\right\rangle$, where $\hat{H}(\mathbf{k})$ is the crystal Hamiltonian acting on $\left|u_{n}(\mathbf{k})\right\rangle$. The orbital magnetization $(\mathrm{OM})$ is then given by the field derivative at fixed temperature and chemical potential, $\mathcal{M}(\mathbf{r})=-(\partial F / \partial \mathbf{B})_{\mu, T}$, with the result

$$
\begin{aligned}
\mathcal{M}(\mathbf{r}) & =\sum_{n} \int d^{3} \mathbf{k m}_{n}(\mathbf{k}) f_{n}(\mathbf{r}, \mathbf{k}) \\
& +\frac{1}{\beta} \sum_{n} \int d^{3} \mathbf{k} \frac{e}{\hbar} \boldsymbol{\Omega}_{n} \ln \left[1+e^{\beta\left(\mu-\varepsilon_{n}^{M}\right)}\right] \\
& \equiv \mathbf{M}_{c}+\mathbf{M}_{\boldsymbol{\Omega}},
\end{aligned}
$$

where $f_{n}(\mathbf{r}, \mathbf{k})$ is the local equilibrium Fermi-Dirac distribution function for $n$-th band. The first term in Eq. (2) is just a statistical sum of the orbital magnetic moments of the carriers originating from the self-rotation of the carrier wave packet ${ }^{3.4}$, thus we call this term the conventional part $M_{c}$ of the OM. Whereas the second term $M_{\Omega}$ is the Berry phase correction to the OM. This term is of topological nature, arising from a bulk consideration. Interestingly, it is this Berry-phase term that eventually enters the transport current ${ }^{2}$. At zero temperature and magnetic field the general expression (2) reduces to 1.5

$$
\mathcal{M}=\sum_{n} \int^{\mu_{0}} d^{3} \mathbf{k}\left(\mathbf{m}_{n}(\mathbf{k})+\frac{e}{\hbar} \boldsymbol{\Omega}_{n}(\mathbf{k})\left[\mu_{0}-\varepsilon_{n \mathbf{k}}\right]\right),
$$

where the upper limit means that the integral is over states with energies below the Fermi energy $\mu_{0}$. As mentioned above, the first term in Eq. (3) is the contribution from the intrinsic orbital moment, and the second term comes from the Berry-phase correction of the density of states.

Among very few studies on Berry phase effect of the $\mathrm{OM}$, Xiao et al. $\stackrel{2}{2}$ have investigated the anomalous thermoelectric transport in $\mathrm{CuCr}_{2} \mathrm{Se}_{4-x} \mathrm{Br}_{x}$ system ${ }^{6}$ by use of their derived relation between anomalous Nernst effect (ANE) and anomalous Hall effect (AHE). Ceresoli et al $^{7}$ have given an extensive tight-binding calculation of OM for finite and periodic two-dimensional (2D) Hal-

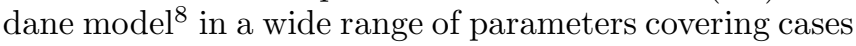


of Chern number (a dimensionless integer ${ }^{\underline{9}}$ ) $C=0$ and $C \neq 0$. Due to its basic importance in understanding the magnetism and transport features of the materials, obviously, more work are needed in exploiting the properties of the OM in various kinds of realistic physical systems. Also the theory itself, such as a full quantum mechanical derivation of the OM, remains to be further developed.

In this paper we extend the study of the OM to the strongly correlated electronic systems. More specificially, we focus our attention to a typical flat-band ferromagnet with spin anisotropies on the 2D kagomé lattice. This attention is partially motivated by the recently established common point that the spin Berry phase plays an important role in the quantum transport in spin-orbit coupled ${ }^{10,11,12}$ or spin-chiral ${ }^{13,14,15,16}$ ferromagnetic systems; the latter is exampled by pyrochlore compounds $\mathrm{R}_{2} \mathrm{Mo}_{2} \mathrm{O}_{7}(\mathrm{R}=\mathrm{Nd}, \mathrm{Sm}, \mathrm{Gd})$, in which the spin configuration is noncoplanar and the spin chirality appears. As a consequence, the quantum transport of electrons, especially the transverse conductivity $\sigma_{x y}$ is expected to be affected by the presence of spin chirality. In fact, recent transport experiments on ferromagnetic pyrochlores have revealed that the AHE increases as the temperature $T$ is lowered and approaches to the saturated value $\underline{17,18}$. This behavior is intrinsically different from the conventional theories 19 . One explanation to this anomalous feature is that the pyrochlore structure has geometrical frustration 20 which consists of cornersharing tetrahedrons. Thus the antiferromagnetic and even the ferromagnetio $\stackrel{21}{ }$ interaction between nearestneighbor spins are frustrated. Recently, Ohgushi et al ${ }^{22}$ have first pointed out that the chiral spin state can be realized by the introduction of spin anisotropy in an ordered spin system on the 2D kagomé lattice, which is the cross section of the pyrochlore lattice perpendicular to the $(1,1,1)$ direction ${ }^{20}$. In this case, it has been shown ${ }^{22}$ that the presence of chiral spin state may induce nonzero Chern number, thus resulting in a quantized Hall effect in insulating state, which is expected to have important implications to AHE experiments in ferromagnetic pyrochlores.

Motivated by the above work ${ }^{22}$ on quantized Hall effect in the 2D kagomé lattice, in this paper we turn to study the OM properties and effects in this 2D lattice system with nonzero Chern number. We show that the OM displays different behavior in the metallic and insu- lating regions, which is due to the different roles $M_{c}$ and $M_{\Omega}$ play in these two regions. Also, the ANE and the orbital magnetic susceptibility are investigated, which further illustrate the fundamental role brought forth by the Berry-phase contribution and nonzero Chern number.

\section{THEORETICAL MODEL AND CHERN NUMBER ANALYSIS}

Now we consider the double-exchange ferromagnet on the kagomé lattice ${ }^{22}$ schematically shown in Fig. 1. Here the triangle is the one face of the tetrahedron, and the easy axis of the spin anisotropy points to the center of each tetrahedron and has an out-of-plane component. In this situation the three local spins on sites A, $\mathrm{B}$, and $\mathrm{C}$ in Fig. 1 have different directions and the spin chirality emerges. The effective Hamiltonian for the hopping electrons strongly Hund-coupled to these localized spins is given by $H=\sum_{N N} t_{i j}^{\text {eff }} \psi_{i}^{\dagger} \psi_{j}$ with $t_{i j}^{\text {eff }}=$ $t\left\langle\chi_{i} \mid \chi_{j}\right\rangle=t e^{i a_{i j}} \cos \frac{\vartheta_{i j}}{2}$. Here the spin wave function $\left|\chi_{i}\right\rangle$ is explicitly given by $\left|\chi_{i}\right\rangle=\left[\cos \frac{\vartheta_{i}}{2}, e^{i \phi_{i}} \sin \frac{\vartheta_{i}}{2}\right]^{\mathrm{T}}$, where the polar coordinates are pinned by the local spins, i.e., $\left\langle\chi_{i}\left|\mathbf{S}_{i}\right| \chi_{i}\right\rangle=\frac{1}{2}\left(\sin \vartheta_{i} \cos \phi_{i}, \sin \vartheta_{i} \sin \phi_{i}, \cos \vartheta_{i}\right) . \vartheta_{i j}$ is the angle between the two spins $\mathbf{S}_{i}$ and $\mathbf{S}_{j}$. The phase factor $a_{i j}$ can be regarded as the gauge vector potential $a_{\mu}(\mathbf{r})$, and the corresponding gauge flux is related to scalar spin chirality $\chi_{i j k}=\mathbf{S}_{i} \cdot\left(\mathbf{S}_{j} \times \mathbf{S}_{k}\right)^{23}$. In periodic crystal lattices, the nonvanishing of the gauge flux relies on the multiband structure with each band being characterized by a Chern number ${ }^{9.24}$. Here the Chern number appears as a result of the spin chirality in ferromagnets. Following Ref ${ }^{22}$ we set the flux originated from the spin chirality per triangle (see Fig. 1) as $\phi$, which satisfies $e^{i \phi}=e^{i\left(a_{A B}+a_{B C}+a_{C A}\right)}$. The flux penetrating one hexagon in Fig. 1 is determined as $-2 \phi$. We take the gauge, in which the phase of $t_{i j}^{e f f}$ is the same for all the nearest-neighbor pairs with the direction shown by the arrows in Fig. 1. It should be pointed out that the net flux through a unit cell vanishes due to the cancelation of the contribution of the two triangles and a hexagon. Also noted is that the time-reversal symmetry is broken except for cases of $\phi=0, \pi$.

The momentum-transformation of the above tightbinding Hamiltonian is given by ${ }^{22}$

$$
H(\mathbf{k})=\left(\begin{array}{ccc}
0 & 2 \cos \left(\mathbf{k} \cdot \mathbf{a}_{1}\right) e^{-i \phi / 3} & 2 \cos \left(\mathbf{k} \cdot \mathbf{a}_{3}\right) e^{i \phi / 3} \\
2 \cos \left(\mathbf{k} \cdot \mathbf{a}_{1}\right) e^{i \phi / 3} & 0 & 2 \cos \left(\mathbf{k} \cdot \mathbf{a}_{2}\right) e^{-i \phi / 3} \\
2 \cos \left(\mathbf{k} \cdot \mathbf{a}_{3}\right) e^{-i \phi / 3} & 2 \cos \left(\mathbf{k} \cdot \mathbf{a}_{2}\right) e^{i \phi / 3} & 0
\end{array}\right)
$$

where $\mathbf{a}_{1}=(-1 / 2,-\sqrt{3} / 2), \quad \mathbf{a}_{2}=(1,0), \quad$ and $\quad \mathbf{a}_{3}=$ $(-1 / 2, \sqrt{3} / 2)$ represent the displacements in a unit cell from $\mathrm{A}$ to $\mathrm{B}$ site, from $\mathrm{B}$ to $\mathrm{C}$ site, and from $\mathrm{C}$ to $\mathrm{A}$ site respectively. In this notation, the Brillouin zone (BZ) is a 


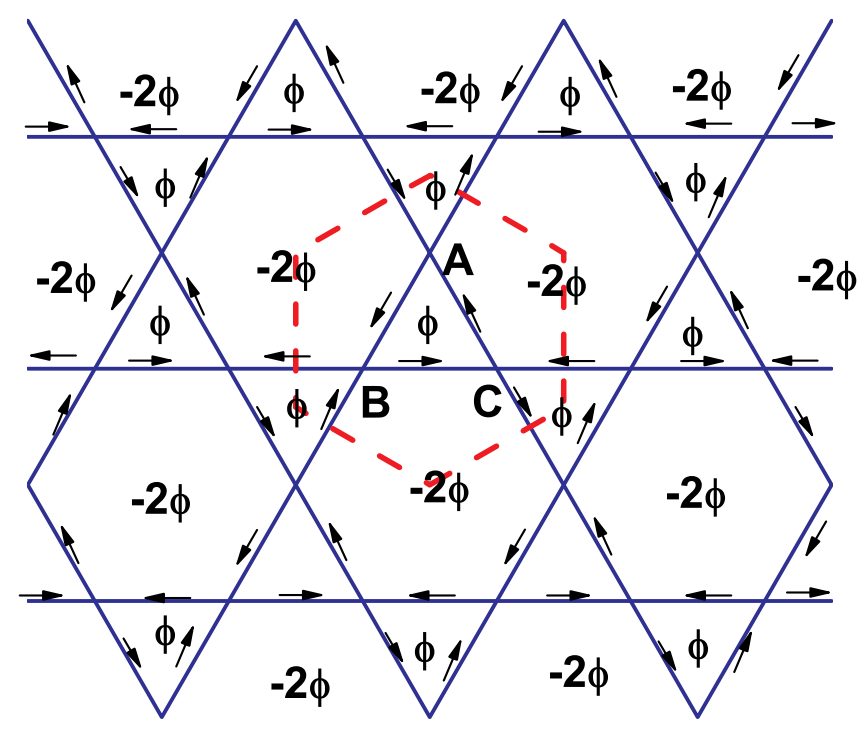

FIG. 1: (Color online). Two dimensional spin-chiral ferromagnetic kagomé lattice. The dashed line represents the Wigner-Seitz unit cell, which contains three independent sites (A, B, C). It is assumed that each site has a different spin anisotropy axis. The arrows means the sign of the phase of the transfer integral $t_{i j}$. hexagon with the corners of $\mathbf{k}= \pm(2 \pi / 3) \mathbf{a}_{1}, \pm(2 \pi / 3) \mathbf{a}_{2}$, $\pm(2 \pi / 3) \mathbf{a}_{3}$, two of which are independent. Note that in writing the Hamiltonian (4) we have chosen the unit of $t \cos \left(\vartheta_{i j} / 2\right)=1$, and set the length of each bond as unity. The eigenenergies of the Hamiltonian (4) are given by

$$
\begin{aligned}
& \varepsilon_{1 \mathbf{k}}=4 \sqrt{\frac{b(\mathbf{k})}{3}} \cos \frac{\theta(\mathbf{k})+2 \pi}{3} \\
& \varepsilon_{2 \mathbf{k}}=4 \sqrt{\frac{b(\mathbf{k})}{3}} \cos \frac{\theta(\mathbf{k})-2 \pi}{3} \\
& \varepsilon_{3 \mathbf{k}}=4 \sqrt{\frac{b(\mathbf{k})}{3}} \cos \frac{\theta(\mathbf{k})}{3}
\end{aligned}
$$

where $b(\mathbf{k})=\cos ^{2}\left(\mathbf{k} \cdot \mathbf{a}_{1}\right)+\cos ^{2}\left(\mathbf{k} \cdot \mathbf{a}_{2}\right)+\cos ^{2}\left(\mathbf{k} \cdot \mathbf{a}_{3}\right)$, $\theta(\mathbf{k})=\arg \left[\sqrt{\frac{27}{b^{3}(\mathbf{k})}} c(\mathbf{k})+i \sqrt{1-\frac{27 c^{2}(\mathbf{k})}{b^{3}(\mathbf{k})}}\right]$, and $c(\mathbf{k})=\cos (\mathbf{k} \cdot$ $\left.\mathbf{a}_{1}\right) \cos \left(\mathbf{k} \cdot \mathbf{a}_{2}\right) \cos \left(\mathbf{k} \cdot \mathbf{a}_{3}\right) \cos \phi$. Because the relation $\varepsilon_{1 \mathbf{k}} \leq \varepsilon_{2 \mathbf{k}} \leq \varepsilon_{3 \mathbf{k}}$ is always satisfied, henceforth we will call these energy bands 'lower', 'middle', and 'upper' bands respectively. The corresponding eigenvectors are

$$
\left|u_{n \mathbf{k}}\right\rangle=G_{n}(\mathbf{k})\left(\begin{array}{c}
\frac{1}{2}\left[\varepsilon_{n \mathbf{k}}^{2}-4 \cos ^{2}\left(\mathbf{k} \cdot \mathbf{a}_{2}\right)\right] \\
e^{i \frac{\phi}{3}}\left[\varepsilon_{n \mathbf{k}} \cos \left(\mathbf{k} \cdot \mathbf{a}_{1}\right)+2 \cos \left(\mathbf{k} \cdot \mathbf{a}_{2}\right) \cos \left(\mathbf{k} \cdot \mathbf{a}_{3}\right) e^{-i \phi}\right] \\
e^{-i \frac{\phi}{3}}\left[\varepsilon_{n \mathbf{k}} \cos \left(\mathbf{k} \cdot \mathbf{a}_{3}\right)+2 \cos \left(\mathbf{k} \cdot \mathbf{a}_{1}\right) \cos \left(\mathbf{k} \cdot \mathbf{a}_{2}\right) e^{-i \phi}\right]
\end{array}\right)
$$

where the normalized factors are given by

$$
G_{n}(\mathbf{k})=\frac{1}{\sqrt{2 b(\mathbf{k}) \varepsilon_{n \mathbf{k}}^{2}+\left(4 b(\mathbf{k})-3 \varepsilon_{n \mathbf{k}}^{2}\right) \cos ^{2}\left(\mathbf{k} \cdot \mathbf{a}_{2}\right)+12 c(\mathbf{k}) \varepsilon_{n \mathbf{k}}}}
$$

First let us see the Chern number and Hall conductivity of this system, which has been briefly reported in Ref $^{22}$. It is clear that the Hall conductivity $\sigma_{x y}$ is equal to zero in the time-reversal symmetric cases $\phi=0, \pi$. Therefore, we focus on the case of $\phi \neq 0, \pi$. In this situation there is an energy gap between the two nearestneighbor bands. We assume that the Fermi energy is lying in the gap. Then the Hall conductivity is a sum over occupied Bloch bands, $\sigma_{x y}=\left(e^{2} / h\right) \sum_{n}^{\text {occu }} C_{n}$, where the $n$-th band Chern number is defined by

$$
\begin{aligned}
C_{n} & =-\frac{1}{2 \pi} \int_{\mathrm{BZ}} d^{2} \mathbf{k} \Omega_{n}(\mathbf{k}) \\
& =-\frac{1}{2 \pi} \int_{\mathrm{BZ}} d^{2} \mathbf{k} \hat{z} \cdot\left(\nabla_{\mathbf{k}} \times \mathbf{A}_{n}(\mathbf{k})\right),
\end{aligned}
$$

where $\mathbf{A}_{n}(\mathbf{k})=i\left\langle u_{n}(\mathbf{k}) \mid \nabla_{\mathbf{k}} u_{n}(\mathbf{k})\right\rangle$ is the Berry phase connection (vector potential) for the $n$-th band. According to the expressions for $\left|u_{n \mathbf{k}}\right\rangle$, one obtains the expression 
for $\mathbf{A}_{n}(\mathbf{k})$ as follows

$\mathbf{A}_{n}(\mathbf{k})=2 \varepsilon_{n \mathbf{k}} G_{n}^{2}(\mathbf{k}) \sin \phi \cos k_{x}\left[\sin \sqrt{3} k_{y} \hat{\mathbf{x}}+\sqrt{3} \sin k_{x} \hat{\mathbf{y}}\right]$.

To proceed with Eqs. (8)-(9), one may first transform the integral of the curl of the vector potential $\mathbf{A}_{n}$ over BZ to the line intergal of $\mathbf{A}_{n}$ along the BZ boundary by use of Stokes' theorem, and then apply the complex contour integration technique and residue theorem to sinusoidal functions. After a straightforward derivation, one obtains $C_{1}=-\operatorname{sgn}(\sin \phi), C_{2}=0$, and $C_{3}=\operatorname{sgn}(\sin \phi)$, respectively, which means that the quantum Hall effect is realized. However, this purely mathematical calculation of Chern number is not favored by theoretical physicists, who instead resort to the physical connotation that the vector potential $\mathbf{A}_{n}$ and gauge flux $\boldsymbol{\Omega}_{n}$ are endowed with. Correspondingly, we start this gauge-field analysis with the notation that the value of Chern number is invariant under gauge transformation $\left|u_{n \mathbf{k}}^{\prime}\right\rangle=e^{i g_{n}(\mathbf{k})}\left|u_{n \mathbf{k}}\right\rangle$, $\mathbf{A}_{n}^{\prime}(\mathbf{k})=\mathbf{A}_{n}(\mathbf{k})-\nabla_{\mathbf{k}} g_{n}(\mathbf{k})$, where $g_{n}(\mathbf{k})$ is an arbitrary smooth function of $\mathbf{k}$. The gauge of the wave functions Eq. (6) has been chosen in such way that the first component is real. If this gauge choice is applicable in the whole region of the BZ, the Chern number will obviously be zero. However, at two equivalent BZ edge points $\mathbf{k}_{0}=(0, \pm \pi / \sqrt{3})$ on BZ boundary, one can find that the wave functions $\left|u_{n \mathbf{k}}\right\rangle$ in Eq. (6) is ill-defined since both its denominator and numerator are zero at these two points. This means that the used gauge cannot apply to the whole BZ and one needs to render a gauge transformation to avoid the singularity at $\mathbf{k}_{0}$. For this one writes down the other set of eigenvectors as follows

$$
\left|u_{n \mathbf{k}}^{\prime}\right\rangle=G_{n}^{\prime}(\mathbf{k})\left(\begin{array}{c}
e^{-i \frac{\phi}{3}}\left[\varepsilon_{n \mathbf{k}} \cos \left(\mathbf{k} \cdot \mathbf{a}_{1}\right)+2 \cos \left(\mathbf{k} \cdot \mathbf{a}_{2}\right) \cos \left(\mathbf{k} \cdot \mathbf{a}_{3}\right) e^{i \phi}\right] \\
\frac{1}{2}\left[\varepsilon_{n \mathbf{k}}^{2}-4 \cos ^{2}\left(\mathbf{k} \cdot \mathbf{a}_{3}\right)\right] \\
e^{i \frac{\phi}{3}}\left[\varepsilon_{n \mathbf{k}} \cos \left(\mathbf{k} \cdot \mathbf{a}_{2}\right)+2 \cos \left(\mathbf{k} \cdot \mathbf{a}_{1}\right) \cos \left(\mathbf{k} \cdot \mathbf{a}_{3}\right) e^{i \phi}\right]
\end{array}\right)
$$

where the second component turns to be real. The nor- malized factors are given by

$$
G_{n}^{\prime}(\mathbf{k})=\frac{1}{\sqrt{2 b(\mathbf{k}) \varepsilon_{n \mathbf{k}}^{2}+\left(4 b(\mathbf{k})-3 \varepsilon_{n \mathbf{k}}^{2}\right) \cos ^{2}\left(\mathbf{k} \cdot \mathbf{a}_{3}\right)+12 c(\mathbf{k}) \varepsilon_{n \mathbf{k}}}} .
$$

The new eigenvectors $\left|u_{n \mathbf{k}}^{\prime}\right\rangle$ recovers the well-defined behavior at $\mathbf{k}_{0}$; the new singular points brought force by $\left|u_{n \mathbf{k}}^{\prime}\right\rangle$ are $\mathbf{k}_{0}^{\prime}= \pm\left(\frac{\pi}{2}, \frac{\pi}{2 \sqrt{3}}\right)$. Thus according to the two different gauge choices, the BZ is now divided into two regions $\mathrm{V}$ and $\mathrm{V}^{\prime}$ as shown in Fig. 2. The wave functions $\left|u_{n \mathbf{k}}\right\rangle$ are used onto the region $\mathrm{V}$, while $\left|u_{n \mathbf{k}}^{\prime}\right\rangle$ apply to $\mathrm{V}^{\prime}$. Note that there remains some freedom in the divi- sion of the BZ. Because $\left|u_{n \mathbf{k}}\right\rangle$ and $\left|u_{n \mathbf{k}}^{\prime}\right\rangle$ are ill-defined only at $\mathbf{k}_{0}$ and $\mathbf{k}_{0}^{\prime}$, respectively, we are free to deform this division as long as $\mathbf{k}_{0}\left(\mathbf{k}_{0}^{\prime}\right) \notin \mathrm{V}\left(\mathrm{V}^{\prime}\right)$. This corresponds

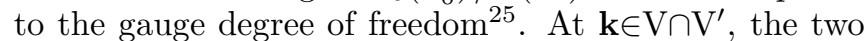
choices of wave functions are different by a phase factor $\left|u_{n \mathbf{k}}^{\prime}\right\rangle=e^{i g_{n}(\mathbf{k})}\left|u_{n \mathbf{k}}\right\rangle$, i.e., $\mathbf{A}_{n}^{\prime}(\mathbf{k})=\mathbf{A}_{n}(\mathbf{k})-\nabla_{\mathbf{k}} g_{n}(\mathbf{k})$, where

$$
e^{i g_{n}(\mathbf{k})}=\frac{G_{n}^{\prime}(\mathbf{k})}{G_{n}(\mathbf{k})} \frac{e^{-i \frac{\phi}{3}}\left[\varepsilon_{n \mathbf{k}} \cos \left(\mathbf{k} \cdot \mathbf{a}_{1}\right)+2 \cos \left(\mathbf{k} \cdot \mathbf{a}_{2}\right) \cos \left(\mathbf{k} \cdot \mathbf{a}_{3}\right) e^{i \phi}\right]}{\frac{1}{2}\left[\varepsilon_{n \mathbf{k}}^{2}-4 \cos ^{2}\left(\mathbf{k} \cdot \mathbf{a}_{2}\right)\right]} .
$$

Thus one obtains the values of nonzero Chern number

for lower and upper bands as follows

$$
\begin{aligned}
C_{n} & =-\frac{1}{2 \pi} \oint_{\partial \mathrm{V}}\left[\mathbf{A}_{n}(\mathbf{k})-\mathbf{A}_{n}^{\prime}(\mathbf{k})\right] \cdot d \mathbf{k}=-\frac{1}{2 \pi} \oint_{\partial \mathrm{V}} d g_{n}(\mathbf{k}) \\
& = \begin{cases}-\operatorname{sgn}(\sin \phi) & \text { (lower band) } \\
\operatorname{sgn}(\sin \phi) & \text { (upper band) }\end{cases}
\end{aligned}
$$



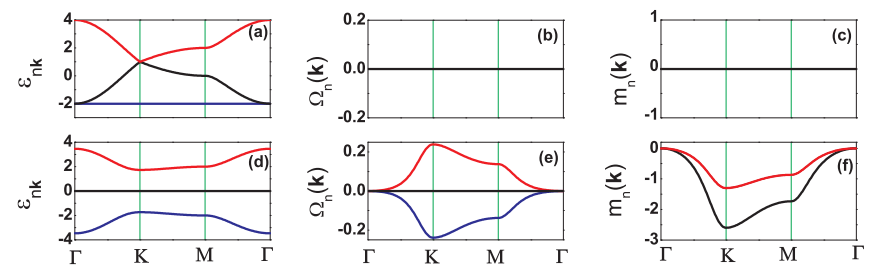

FIG. 2: (Color online). Division of the Brillouin zone of the kagomé lattice model into two regions $\mathrm{V}$ (red area) and $\mathrm{V}^{\prime}$ (blue area).

which is confirmed by the explicit calculation based on the complex-contour integration technique. The nonzero Chern number implies that the wave function cannot be written as a single function for the entire BZ. This also affects the definition of field operators $a_{n \mathbf{k}} 25$. Let $a_{n \mathbf{k}}$ denote the annihilation operators when $\mathbf{k} \in \mathrm{V}$. Then $\left|u_{n \mathbf{k}}^{\prime}\right\rangle=e^{i g_{n}(\mathbf{k})}\left|u_{n \mathbf{k}}\right\rangle$ implies the field operator to be $e^{-i g_{n}(\mathbf{k})} a_{n \mathbf{k}}$ at $\mathbf{k} \in \mathrm{V}^{\prime}$. This phase mismatch between patches in the BZ provides the quantized Hall conductivity.

\section{ORBITAL MAGNETIZATION AND ITS EFFECTS}

Now we turn to study the OM and its various effects. In the present two-dimensional case, the magnetization and Berry curvature become pseudoscalar quantities $M_{c}$, $M_{\Omega}$, and $\Omega_{n}$. From Eq. (3) one can see that for band insulator there will be a discontinuity in $\mathrm{OM}$ if the integral of the Berry curvature over the entire BZ, or the anomalous Hall conductivity, is nonzero and quantized. The size of the discontinuity is given by the quantized anomalous Hall conductivity times $E_{g} / e$, where $E_{g}$ is the energy gap. Because an analytic derivation of the OM for 2D kagomé lattice is very tedious, thus we turn instead to a full numerical representation in terms of the eigenstates $\left|u_{n \mathbf{k}}\right\rangle$ and eigenenergies $\varepsilon_{n \mathbf{k}}$ given in Eqs. (5)-(6).

We first show in Figs. 3 structures of the energy bands $\varepsilon_{n \mathbf{k}}$, the crystal orbital moment $m_{n}(\mathbf{k})$, and the Berry curvature $\Omega_{n}(\mathbf{k})$ along high-symmetry lines in the BZ for the values of $\phi=0$ (upper panels) and $\phi=\pi / 2$ (lower panels). In the case of $\phi=0$, the time-reversal symmetry is preserved. From Fig. 3(a) one can see that for $\phi=0$ the middle band and upper band touch at the corner points (denoted by $\mathbf{k}_{\mathrm{K}}$ ) in the $\mathrm{BZ}$, around which the upper and middle bands exhibit a cusp, $\epsilon_{3 \mathbf{k}}$ $\left(\epsilon_{2 \mathbf{k}}\right)=1 \pm \sqrt{3}\left|\mathbf{k}-\mathbf{k}_{\mathrm{K}}\right|$. In this case, the low-energy quasiparticle excitations can be well understood within a $(2+1)-D$ Dirac fermion field theory. The lower band becomes dispersionless $\left(\varepsilon_{1 \mathbf{k}}=-2\right)$ at $\phi=0$, which reflects the fact that the $2 \mathrm{D}$ kagomé lattice is a line graph of the honeycomb structure $\underline{26}$. This flat band touches at the $\Gamma$ point of the $\mathrm{BZ}$ with the middle band, whose dispersion around $\Gamma$ looks like an isotropic paraboloid,

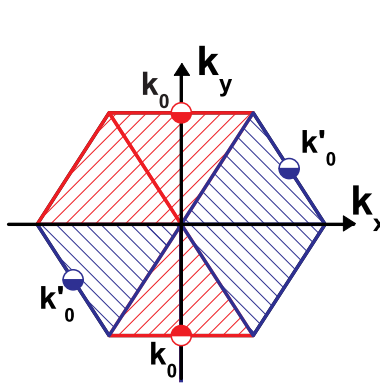

(a)

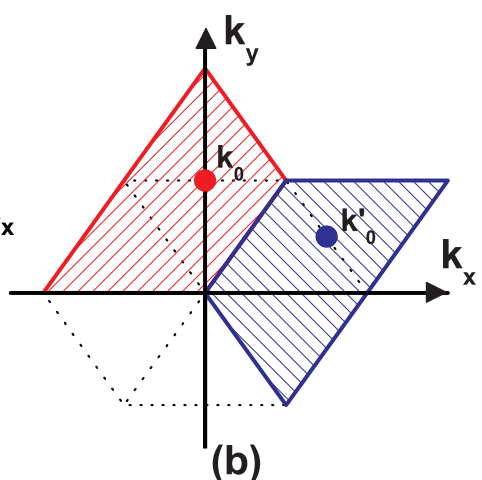

(b)
FIG. 3: (Color online). (From left to right) band strcture, Berry curvature $\Omega_{n}(\mathbf{k})$, and conventional orbital magnetic moment $m_{n}(\mathbf{k})$. The blue, black, and red curves correspond to the lower, middel, and upper bands respectively. The spin chirality parameter is chosen to be $\phi=0$ for upper panels while $\phi=\pi / 2$ for lower panels.

$\varepsilon_{2 \mathbf{k}}=-2+\frac{1}{8} k^{2}$. Note that the energy spectrum for $\phi=\pi$ (not shown here) is a particle-hole conjugate of that for $\phi=0$; therefore, the upper band becomes flat with an eigenvalue of 2 . The Berry curvature $\Omega_{n}(\mathbf{k})$ and the intrinsic orbital magnetic moment $m_{n}(\mathbf{k})$ for three bands are zero everywhere in the whole $\mathrm{BZ}$ in the time-reversal symmetric case of $\phi=0$, as shown in Figs. 3(b)-(c). This can be simply seen by the fact that the wave function $\left|u_{n \mathbf{k}}\right\rangle$ for $\phi=0$ is real. Generally they satisfy the property $m_{n}(-\mathbf{k})=-m_{n}(\mathbf{k}), \Omega_{n}(-\mathbf{k})=-\Omega_{n}(\mathbf{k})$ under timereversal symmetry and $m_{n}(-\mathbf{k})=m_{n}(\mathbf{k}), \Omega_{n}(-\mathbf{k})=\Omega_{n}(\mathbf{k})$ under spatial inversion symmetry. Thus in a crystal with both time-reversal symmetry and spatial inversion symmetry, $\boldsymbol{\Omega}_{n}(\mathbf{k})$ and $\mathbf{m}_{n}(\mathbf{k})$ will disappear in the BZ. Figures 3(d)-(f) show $\varepsilon_{n \mathbf{k}}, \Omega_{n}(\mathbf{k})$, and $m_{n}(\mathbf{k})$ for the value of $\phi=\pi / 2$. In this case the time-reversal symmetry is broken. One can see that there does no longer exist degeneracy in the energy spectrum and two gaps among three bands now open. The middle band turns to become flat for $\phi=\pi / 2$, and the energy spectra shows a particle-hole symmetry. Note that generally the energy spectra has no particle-hole symmetry except for the cases of $\phi= \pm \pi / 2$. This particle-hole symmetry at $\phi=\pi / 2$ is also reflected in $\Omega_{n}(\mathbf{k})$ [Fig. 3(e)] through the feature that the Berry curvature for the flat middle band is zero everywhere in the BZ, while the Berry curvatures for lower and upper bands are nonzero and different with a sign. The intrinsic magnetic moment $m_{n}(\mathbf{k})$ for $\phi=\pi / 2$ is shown in Fig. $3(\mathrm{f})$. One can see that unlike the Berry curvature, $m_{2}(\mathbf{k})$ for the middle flat band is nonzero in the $\mathrm{BZ}$ and its amplitude is the sum of $m_{1}(\mathbf{k})$ and $m_{3}(\mathbf{k})$, which are equal in the presence of particle-hole symmetry.

Figure $4(\mathrm{a})$ shows the OM $(\mathcal{M})$ as a function of the electron chemical potential $\mu$ for the value of $\phi=\pi / 3$, in which case neither time-reversal symmetry nor particlehole symmetry is preserved. One can see that initially the OM rapidly decreases as the filling of the lower band 

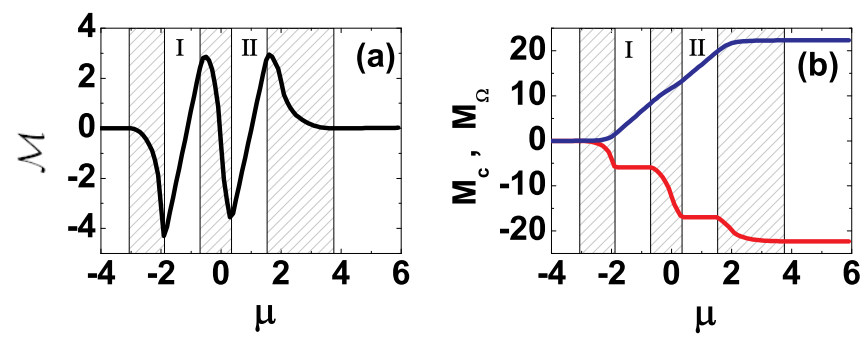

FIG. 4: (Color online). (a) Orbital magnetization of the kagomé lattice, and (b) its two components $M_{c}$ (red curve) and $M_{\Omega}$ (blue curve) as a function of the electron chemical potential $\mu$ for $\phi=\pi / 3$. The shaded areas correspond to the three groups of bands. To suppress the divergence at band/gap contacts, we have used the temperature of $k_{B} T=0.05$.

increases, arriving at a minimum at $\mu=-2$, a value corresponding to the top of the lower band. Then, as the chemical potential continues to vary in the gap [region I in Fig. 4(a)] between the lower band and middle band, the OM goes up and increases as a linear function of $\mu$. This linear relationship in the insulating region can be understood by Eq. (3), from which one obtains

$$
\begin{aligned}
\frac{d \mathcal{M}}{d \mu} & =\frac{e}{\hbar} \sum_{n}^{\text {occu }} \int \frac{d^{2} k}{(2 \pi)^{2}} \Omega_{n}(\mathbf{k}) \\
& =-\frac{e}{h} \sum_{n}^{\text {occu }} C_{n} .
\end{aligned}
$$

Thus when the chemical potential varies in the gap between $\varepsilon_{1 \mathbf{k}}$ and $\varepsilon_{2 \mathbf{k}}$, only the lower band $\varepsilon_{1 \mathbf{k}}$ is occupied and $d \mathcal{M} / d \mu=-(e / h) C_{1}$. For $\phi=\pi / 3, C_{1}=-1$. Thus $d \mathcal{M} / d \mu=e / h$, i.e., the OM linearly increases with the chemical potential in the insulating region I as shown in Fig. 4(a). When the chemical potential touches the bottom of the middle band, then the OM suddenly switches down and rapidly decreases again when the chemical potential goes through the middle band. The turning behavior at the band/gap contacts becomes divergent at $k_{B} T=0$. This discontinuity is due to the singular behavior of $\Omega_{n}(\mathbf{k})$ and $m_{n}(\mathbf{k})$ at the BZ edge points $\mathbf{k}=\mathbf{k}_{0}$, which will play their role when the $k$-integral is over the entire BZ. When the chemical potential lies in the gap between the middle band and upper band, then the OM goes up again and increases linearly with $\mu$ as shown in the insulating region II in Fig. 4(a). Since the Chern number of middle band is zero, thus from Eq. (14) and Fig. 4(a) one can see that the slope of the OM curve in the insulating region II in Fig. 4(a) is the same as that in the insulating region $\mathrm{I}$.

The totally different behavior of the OM in the metallic and insulating regions, as shown in Fig. 4(a), is due to the different roles $M_{c}$ and $M_{\Omega}$ play in these two regions. For further illustration, we show in Fig. 4(b) $M_{c}$ (red curve) and $M_{\Omega}$ (blue curve) as a function of the chemical potential, their sum gives $\mathcal{M}$ in Fig. 4(a). One can see

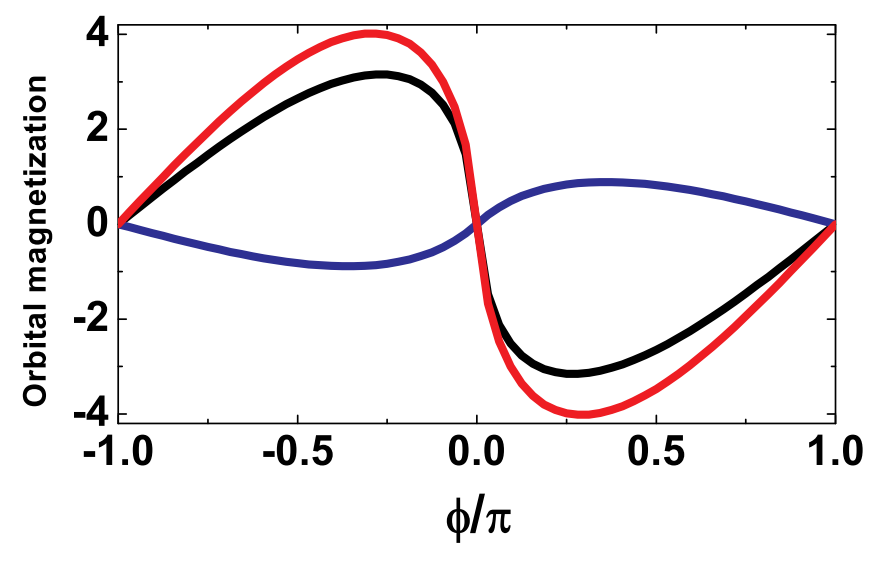

FIG. 5: (Color online). Orbital magnetization (black curve) of the kagomé lattice, and its two components $M_{c}$ (red curve) and $M_{\Omega}$ (blue curve) as a function of the spin chirality parameter $\phi$ for the value of $\mu=-2$, corresponding to the situation that the lower band is partially occupied while the other two bands are empty.

that overall $M_{c}$ and $M_{\Omega}$ have opposite contributions to $\mathcal{M}$, which implies that these two parts carry oppositecirculating currents. In each insulating area the conventional term $M_{c}$ keeps a constant, which is due to the fact that the upper limit of the $k$-integral of $m_{n}(\mathbf{k})$ is invariant as the chemical potential varies in the gap. In the metallic region, however, since the occupied states varies with the chemical potential, thus $M_{c}$ also varies with $\mu$, resulting in a decreasing slope shown in Fig. 4(b). The Berry phase term $M_{\Omega}$ also displays different behavior between insulating and metallic regions. In the insulating region, $M_{\Omega}$ linearly increases with $\mu$, as is expected from Eq. (3). In the metallic region, however, this term sensitively depends on the topological property of the band in which the chemical potential is located. For the lower and upper bands with nonzero Chen number, one can see from Fig. 4(b) that $M_{\Omega}$ remains invariant, while for the middle band of zero Chern number, it increases with the chemical potential $\mu$. On the whole the comparison between Fig. 4(a) and Fig. 4(b) shows that the metallic behavior of $\mathcal{M}$ is dominated by its conventional term $M_{c}$, while in the insulating regime $M_{\Omega}$ plays a main role in determining the behavior of $\mathcal{M}$.

Figure 5 shows the $\mathrm{OM} \mathcal{M}$ (black curve) and its two components $M_{c}$ (red curve) and $M_{\Omega}$ (blue curve) as a function of spin chirality parameter $\phi$ for the value of $\mu=-2$. This value of $\mu$ ensures that the lower band is partially occupied in the whole range of $\phi$. Thus Figure 5 describes the metallic behavior of $\mathcal{M}$. Two prominent features can be observed from Fig. 5: (i) The OM is antisymmetric with respect to $\phi, \mathcal{M}(-\phi)=-\mathcal{M}(\phi)$. This implies opposite circulating currents carried by Bloch states with $\phi$ and those with $-\phi$; (ii) In the metallic situation, one can see that the amplitude of $M_{\Omega}$ is much smaller than that of $M_{c}$. As a consequence, the OM is domi- 
nated by its conventional part $M_{c}$ in the whole range of $\phi$. In the insulating region (not shown in Fig. 5), however, the amplitude of $M_{\Omega}$ largely increases and can be even larger than the conventional contribution for some values of $\phi$ [see Fig. 4].

For transport studies of the OM, here we analyze the properties of ANE in the 2D kagomé lattice. The relation between the OM and ANE has been recently found ${ }^{2}$. To discuss the transport measurement, it is important to discount the contribution from the magnetization current, a point which has attracted much discussion in the past. Cooper et al $\stackrel{27}{\underline{b}}$ have argued that the magnetization current cannot be measured by conventional transport experiments. Xiao et $\mathrm{al}_{\stackrel{2}{2}}^{2}$ have adopted this point and built up a remarkable picture that the conventional orbital magnetic moment $M_{c}$ does not contribute to the transport curent, while the Berry phase term in Eq. (2) directly enters and therefore modifies the intrinsic trans- port Hall current equation as follows

$$
\mathbf{j}_{\mathrm{H}}=-\frac{e^{2}}{\hbar} \mathbf{E} \times \sum_{n} \int \frac{d^{2} k}{(2 \pi)^{2}} f_{n}(\mathbf{r}, \mathbf{k}) \Omega_{n}(\mathbf{k})-\nabla \times \mathbf{M}_{\Omega}(\mathbf{r}),
$$

In the case of uniform temperature and chemical potential, obviously, the second term is zero and the Hall effect of 2D kagomé lattice is featured by nonzero Chern number as discussed by Ohgushi et al $\stackrel{22}{=}$ as well as in this paper. In the following, however, we turn to study another situation, where the current-driving force is not provided by the electric field $(\mathbf{E}=0)$. Instead, it is provided by a statistical force, i.e., the gradient of temperature $T$. In this case, Eqs. (15) and (2) give the expression of intrinsic thermoelectric Hall current as $j_{x}=\alpha_{x y}\left(-\nabla_{y} T\right)$, where the anomalous Nernst conductivity $\alpha_{x y}$ is given by $\underline{2}^{2}$

$$
\alpha_{x y}=\frac{1}{T} \frac{e}{\hbar} \sum_{n} \int \frac{d^{2} k}{(2 \pi)^{2}} \Omega_{n}\left[\left(\epsilon_{n \mathbf{k}}-\mu\right) f_{n}+k_{B} T \ln \left(1+e^{-\beta\left(\epsilon_{n \mathbf{k}}-\mu\right)}\right)\right]
$$

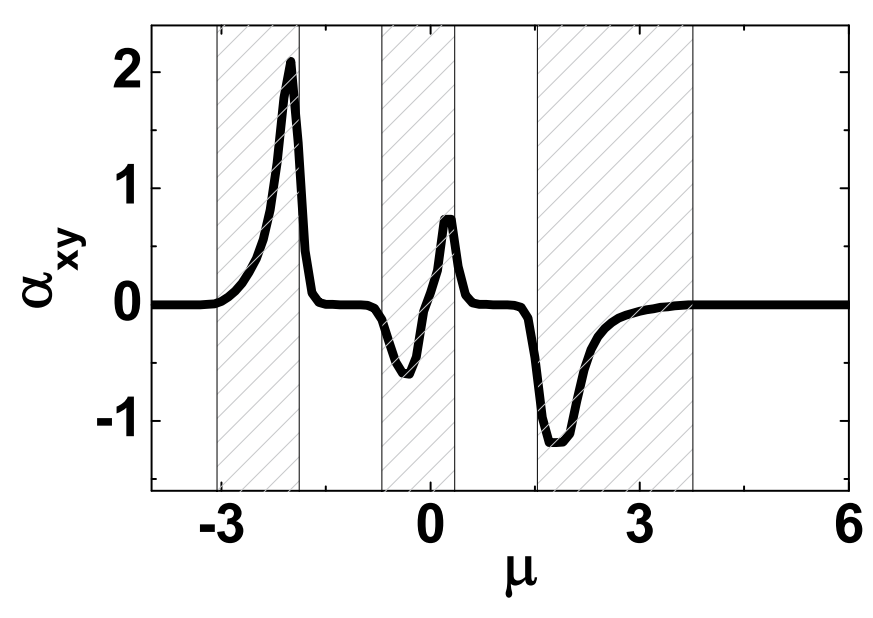

FIG. 6: The intrinsic anomalous Nernst conductivity $\alpha_{x y}$ (divided by the temperature $T$ ) of the kagomé lattice as a function of the electron chemical potential $\mu$ for $\phi=\pi / 3$ and $k_{B} T=0.05$. The shaded areas correspond to the three groups of bands.

Figure 6 shows $\alpha_{x y}$ of the 2D kagomé lattice as a function of the chemical potential for $\phi=\pi / 3$ and $k_{B} T=0.05$. One can see that the ANE disappears in the insulating regions, and when scanning the chemical potential through the bands, there will appear peaks and valleys. Remarkably, a similar peak-valley structure was also found by the recent first-principles calculations in $\mathrm{CuCr}_{2} \mathrm{Se}_{4-x} \mathrm{Br}_{x}$ compound $^{2}$. The ANE of this compound was recently measured by Lee et al $\underline{\underline{6}}$ as a function of $\mathrm{Br}$ doping $x$ which is used to change the chemical potential $\mu$. Due to the scarce data available, until now the peak-valley structure of $\alpha_{x y}$ revealed in Fig. 6 and in Ref $\stackrel{2}{=}$ has not been found in experiment, and more direct experimental results are needed for quantitative comparison with the theoretical results. Interestingly, the expression for $\alpha_{x y}$ can be simplied at low temperature as the Mott relation ${ }^{2}$,

$$
\alpha_{x y}=-\frac{\pi^{2}}{3} \frac{k_{B}^{2} T}{e} \sigma_{x y}^{\prime}\left(\mu_{0}\right),
$$

where $\sigma_{x y}^{\prime}\left(\mu_{0}\right)$ is the derivative of Hall conductivity with respect to the zero-temperature chemical potential (Fermi energy) $\mu_{0}$. Thus one can see that unlike AHE, ANE is given by the Fermi-surface contribution of the band structure and Berry curvature. Another unique feature of $\alpha_{x y}$ is its linear dependence of temperature.

Finally, let us consider the response of the OM to the external magnetic field $B$. It should be noticed that the above semiclassical theory is carried out up to the first order in the external perturbation ${ }^{4}$. Thus our discussion is valid in the weak magnetic field. From Eq. (2) one obtains the magnetic susceptibility of the OM,

$$
\begin{aligned}
\chi_{\mathrm{o}} & =\frac{\partial \mathcal{M}}{\partial B} \\
& =\sum_{n} \int \frac{d^{2} k}{(2 \pi)^{2}}\left[m_{n}^{2}(\mathbf{k}) \frac{\partial f_{n}}{\partial \mu}+\frac{e}{\hbar} \Omega_{n}(\mathbf{k}) m_{n}(\mathbf{k}) f_{n}\right] \\
& \equiv \chi_{\mathrm{o}}^{(c)}+\chi_{\mathrm{o}}^{(\Omega)} .
\end{aligned}
$$

Obviously, the first conventional term $\chi_{\mathrm{o}}^{(c)}$ describes the 

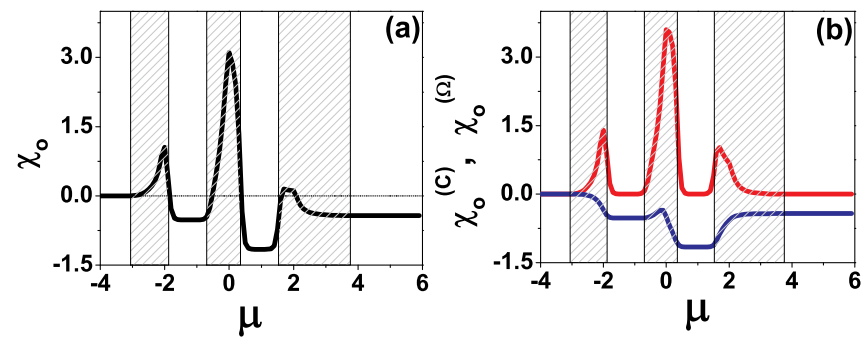

FIG. 7: (Color online). (a) Orbital magnetic susceptibility $\chi_{\mathrm{o}}$ of the kagomé lattice, and (b) its two components contributed from $M_{c}$ (red curve) and $M_{\Omega}$ (blue curve) as a function of the electron chemical potential $\mu$ for $\phi=\pi / 3$ and $k_{B} T=0.05$.

Fermi-surface contribution to $\chi_{\mathrm{o}}$ and thus will disappear in the insulating region. Whereas the second term $\chi_{0}^{(\Omega)}$ denotes the Berry phase correction. Clearly, to calculate $\chi_{\mathrm{o}}^{(\Omega)}$ all the occupied states within the Fermi level should be taken into account. Therefore, the Berry phase correction will give a fundamental contribution to $\chi_{\mathrm{o}}$ in the insulating region for the systems with nonzero Chern number.

Figures 7(a)-(b) show the orbital magnetic susceptibility and its two components as a function of the chemical potential, respectively. From Fig. 7(b) one can see that the conventional part $\chi_{\mathrm{o}}^{(c)}$ always displays the paramagnetic property, while the Berry phase correction contributes a diamagnetic response to $B$. Again, in the metallic regions, the total magnetic susceptibility is dominated by its conventional part and thus displays the paramagnetic feature. Whereas in the insulating regions the nonzero $\chi_{\mathrm{o}}$ comes solely from the Berry phase correction and displays the diamagnetic feature.

Up to now, we have concentrated on the ferromagnet represented by the double-exchange model. As Ohgushi et al..$^{22}$ have pointed out, the present theory is also applicable to the ferromagnet based on the Hubbard model. Furthermore, the spin-orbit coupling gives the spin anisotropies, which introduces the tilting of the spins from the perfect ferromagnetic alignment as assumed in Eq. (4). The present 2D kagomé lattice may be relevant to the recent experiments on pyrochlore compounds $\mathrm{R}_{2} \mathrm{Mo}_{2} \mathrm{O}_{7}(\mathrm{R}=\mathrm{Nd}, \mathrm{Sm}, \mathrm{Gd})^{17}, 18$, which are itinerant ferromagnets on the verge of a Mott transition on the pyrochlore lattice. It can be expected that easy-axis spin anisotropy in these compounds produces the spin chirality by the symmetry consideration. Thus the present results of the OM properties may be verified in such systems.

\section{CONCLUSION}

In summary, we have theoretically studied the properties of $\mathrm{OM}$ and its effects in the 2D spin-chiral ferromagnetic kagomé lattice. The spin chirality parameter $\phi$ in the lattice produces nonzero Chern number and results in profound effects on the OM properties. We have found that the two parts $M_{c}$ and $M_{\Omega}$ in OM opposite each other, and yield the paramagnetic and diamagnetic responses respectively. In particular, due to its Chernnumber property, the magnetic susceptibility of $M_{\Omega}$ remains to be a nonzero constant when the Fermi energy is located in the energy gap of the kagomé lattice. It has been further shown that the OM displays fully different behavior in the metallic and insulating regions, because of different roles $M_{c}$ and $M_{\Omega}$ play in these two regions. The anomalous Nernst conductivity has also been studied, which displays a peak-valley structure as a function of the electron chemical potential. The experiments on ferromagnets are urgently expected to realize these interesting theoretical results.

\section{Acknowledgments}

This work was supported by CNSF under Grant No. 10544004 and 10604010.
1 D. Xiao, J. Shi, and Q. Niu, Phys. Rev. Lett. 95, 137204 (2005).

2 D. Xiao, Y. Yao, Z. Fang, and Q. Niu, Phys. Rev. Lett. 97, 026603 (2006).

3 M.-C. Chang and Q. Niu, Phys. Rev. B 53, 7010 (1996).

${ }^{4}$ G. Sundaram and Q. Niu, Phys. Rev. B 59, 14915 (1999).

5 T. Thonhauser, D. Ceresoli, D. Vanderbilt, and R. Resta, Phys. Rev. Lett. 95, 137205 (2005).

6 W.-L. Lee, S.Watauchi, V.L. Miller, R.J. Cava, and N.P. Ong, Science 303, 1647 (2004); Phys. Rev. Lett. 93, 226601 (2006).

7 D. Ceresoli, T. Thonhauser, D. Vanderbilt, and R. Resta, Phys. Rev. B 74, 024408 (2006).

${ }^{8}$ F.D.M. Haldane, Phys. Rev. Lett. 61, 2015 (1988).

9 D.J. Thouless, Topological Quantum Numbers in Nonrela- tivistic Physics (World Scientific, Singapore, 1998).

10 T. Jungwirth, Q. Niu, and A.H. MacDonald, Phys. Rev. Lett. 88, 207208 (2002).

11 Z. Fang, N. Nagaosa, K.S. Takahashi, A. Asamitsu, R. Mathieu, T. Ogasawara, H. Yamada, M. Kawasaki, Y. Tokura, and K. Terakura, Science 302, 92 (2003).

12 Y. Yao, L. Kleinman, A.H. MacDonald, J. Sinova, T. Jungwirth, D.-S. Wang, E. Wang, and Q. Niu, Phys. Rev. Lett. 92, 037204 (2004).

13 P. Matl et al., Phys. Rev. B 57, 10248 (1998).

14 S. H. Chun et al., Phys. Rev. Lett. 84, 757 (2000).

15 J. Ye et al., Phys. Rev. Lett. 83, 3737 (1999).

16 Y. Taguchi et al., Science 291, 2573 (2001).

17 Y. Taguchi and Y. Tokura, Phys. Rev. B 60, 10280 (1999).

18 T. Katsufuji, H. Y. Hwang, and S-W. Cheong, Phys. Rev. 
Lett. 84, 1998 (2000).

19 C. M. Hurd, The Hall Effect in Metals and Alloys (Plenum Press, New York, 1972).

20 A. P. Ramirez, Annu. Rev. Mater. Sci. 24, 453 (1994); M. J. Harris ans M. P. Zinkin, Mod. Phys. Lett. B 10, 417 (1996).

21 M. J. Harris et al., Phys. Rev. Lett. 79, 2554 (1997); S. T. Bramwell and M. J. Harris, J. Phys.: Condens. Matter 10, L215 (1998); R. Moessner, Phys. Rev. B 57, R5587 (1998).

${ }^{22}$ K. Ohgushi, S. Murakami, and N. Nagaosa, Phys. Rev. B 62, R6065 (2000).

${ }^{23}$ V. Kalmeyer and R. B. Laughlin, Phys. Rev. Lett. 59, 2095 (1987); G. Baskaran and P. W. Anderson, Phys. Rev.
B 37, 580 (1988); R. B. Laughlin, Science 242, 525 (1988); X. G. Wen, F. Wilczek, and A. Zee, Phys. Rev. B 39, 11413 (1989).

24 R. Shindou and N. Nagaosa, Phys. Rev. Lett. 87, 116801 (2001).

25 S. Murakami and N. Nagaosa, Phys. Rev. Lett. 90, 057002 (2003).

26 A. Mielke, J. Phys. A 24, L73 (1991); 24, 3311 (1991); 25, 4335 (1992).

27 N.R. Cooper, B.I. Halperin, and I.M. Ruzin, Phys. Rev. B 55, 2344 (1997). 